\title{
Lipid peroxidation in rats chronically fed ethanol
}

\author{
J P Teare, S M Greenfield, D Watson, N A Punchard, N Miller, C A Rice-Evans, \\ R P H Thompson
}

Gastrointestinal laboratory, The Rayne Institute, St Thomas's Hospital, London J P Teare

$S$ M Greenfield

D Watson

N A Punchard

R P H Thompson

Department of Biochemistry, Guy's Hospital, St Thomas's Street, London $\mathrm{N}$ Miller

C A Rice-Evans

Correspondence to: Dr J P Teare, Liver Unit, Academic Department of Medicine, St Mary's Hospital Medical School, Imperial College of Science, Imperial College of Science, London W2 1 PG

London W2 1PG 22 February 1994

\begin{abstract}
Chronic alcohol consumption induces cytochrome P450IIE1, enabling habitual abusers to consume far greater quantities of alcohol than normal subjects. This pathway of metabolism leads to the production of free radical species, which cause tissue damage through peroxidation of cell membranes. Groups of Wistar rats of equal male: female ratio $(n=24)$ were fed alcohol by gavage twice daily to achieve a dosage of $15 \mathrm{~g} / \mathrm{kg}$ body weight. Mean peak blood alcohol concentrations of $186 \mathbf{~ m g} \%$ were produced in males and $156 \mathrm{mg} \%$ in females. The animals were allowed free access to standard laboratory chow and water. Control animals were pair-fed to the alcoholic group and fed isocaloric glucose by gavage. Groups of animals were killed between 9 and 11 am on consecutive mornings, after nocturnal feeding, since it has previously been shown that fasting rapidly depletes hepatic glutathione concentrations. Hepatic glutathione was measured by a spectrophotometric enzymatic recycling procedure. As a marker of lipid peroxidation hepatic malonaldehyde (MDA) was measured by high performance liquid chromatography. Hepatic MDA was increased in the alcoholic group $(p<0.001)$, as was total hepatic glutathione $(\mathbf{p}<0.0001)$. Plasma concentrations of $\alpha$-tocopherol were increased in the alcoholic group, but ascorbic acid and superoxide dismutase values were not affected. No sex differences were detected. The increased MDA production in the alcohol group is strong evidence that lipid peroxidation is a mechanism of alcoholic tissue damage. The rise in hepatic glutathione may be an adaptive response to free radical production that protects the rat against tissue damage.

(Gut 1994; 35: 1644-1647)
\end{abstract}

At low concentrations most alcohol is metabolised by cytosolic alcohol dehdrogenase to acetaldehyde. Another microsomal ethanol oxidising system, named cytochrome P450 IIE1 requires higher alcohol concentrations for maximal activity, is induced by alcohol consumption, and also leads to the formation of acetaldehyde. Acetaldehyde may be the cause of liver injury by forming adducts with proteins $^{1}$ and inactivating enzymes, or by stimulating the formation of antibodies to these adducts ${ }^{1}$ and hence generating an immune mediated hepatitis. During the metabolism of alcohol to acetaldehyde and the further metabolism of acetaldehyde by cytochrome P450IIE1, toxic free radical metabolites are formed. These cause the lipid peroxidation demonstrable in alcoholic liver disease as either thiobarbituric acid reactive substances $^{2}$ or other aldehydic products, ${ }^{3}$ diene conjugates, ${ }^{4}$ hydroxyl radicals, ${ }^{5}$ superoxide anion, ${ }^{6}$ detected by electron spin traps. ${ }^{78}$ Other authors, however, have refuted these results. ${ }^{910}$

A major antioxidant that is important in preventing free radical mediated lipid peroxidation is the endogenous tripeptide glutathione. Glutathione concentrations are normally lowest in centrilobular hepatocytes where the earliest lesions in alcoholic liver disease are seen, while experimental reduction of hepatic glutathione ${ }^{11}$ increases hepatic damage by ethanol. In man, liver biopsy specimens from chronic alcohol abusers contain lower levels of glutathione. ${ }^{12}$

To determine the precise role of lipid peroxidation in the pathogenesis of alcoholic liver disease, we aimed to produce experimental alcoholic liver disease in Wistar rats, and then to determine the levels of peroxidative end products and of hepatic antioxidants.

\section{Methods}

Groups of Wistar rats of equal male: female sex ratio $(n=24)$ were fed alcohol by $3 \mathrm{ml}$ gavage twice daily. The initial dosage contained $20 \%$ alcohol $/ 80 \%$ dextrose $(277 \mathrm{mmol} / \mathrm{l})$ and this was increased by weekly increments of $10 \%$, increasing the dosage over a four week period to $60 \%$ alcohol to achieve a total daily dosage of $15 \mathrm{~g} / \mathrm{kg}$ body weight. Animals were allowed free access to standard laboratory chow and water. Control animals were pair-fed to the alcoholic group and fed isocaloric dextrose by gavage. The gavage was continued for 12 weeks. After eight weeks, the blood alcohol concentrations were measured by gas liquid chromatography from tail-vein samples taken six hourly over a 24 hour period. Samples were taken from three animals at each time point.

Groups of animals were killed between 9 and 11 am on consecutive mornings, after nocturnal feeding, since we have previously shown that fasting rapidly depletes hepatic glutathione concentrations (unpublished observations).

\section{BIOCHEMICAL ANALYSIS}

\section{Hepatic glutathione}

Within four minutes of death the liver was removed and $1.0 \mathrm{~g}$ homogenised in five volumes of $1 \%$ picric acid. The homogenate 


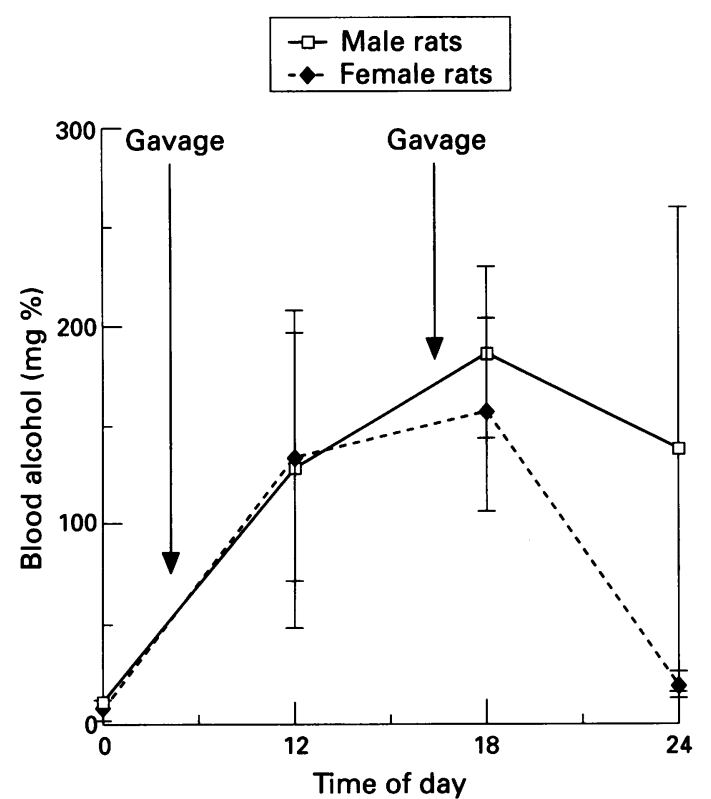

Figure 1: Twenty four hour blood alcohol profiles (values, mean (SEM)).

was then centrifuged $(2500 \mathrm{~g} ; 15$ minutes) and the supernatant stored at $-70^{\circ} \mathrm{C}$ for less than four weeks until later analysis as previously reported. ${ }^{13}$

\section{Hepatic malonaldehyde}

An aliquot of liver was homogenised in five volumes of phosphate buffered saline $(\mathrm{pH} \mathrm{7 \cdot 4)}$ and stored at $-70^{\circ} \mathrm{C}$ for less than four weeks until analysis by high performance liquid chromatography (HPLC) according to Esterbauer. ${ }^{14}$ This technique was chosen as it is more specific than the measurement of thiobarbituric acid reactive material, which also registers decomposition products that arise during the heating stage of the reaction ${ }^{15}$ and non-lipid derived products.

Plasma ascorbic acid

Plasma was treated with trichloroacetic acid (TCA) to obtain a protein free extract, stable when stored at $-70^{\circ} \mathrm{C}$. Ascorbic acid in the deproteinised sample is oxidised by cupric ions to dehydroascorbic acid and diketogulonic acid. These are then reacted with 2,4 dinitrophenylhydrazine ${ }^{16}$ in the presence of sulphuric

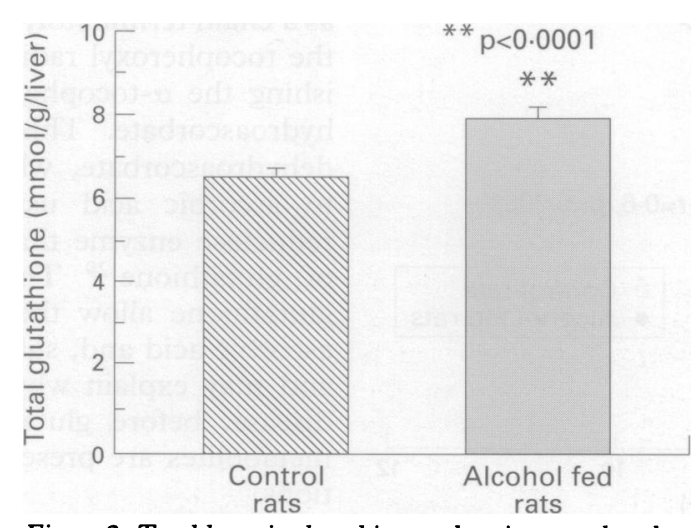

Figure 2: Total hepatic glutathione values in control and alcohol fed rats (values, mean (SEM)). acid to form a coloured phenylhydrazone derivative, which is read at $520 \mathrm{~nm}$. The method thus measures total ascorbate (ascorbic acid and dehydroascorbic acid).

Hepatic Ascorbic acid

An aliquot of liver was homogenised in 10 volumes of $50 \mathrm{~g} / 1 \mathrm{TCA}$ to bring the expected concentration of the vitamin to within the linear range of the assay. The supernatant was taken for vitamin $C$ analysis by the same method as for plasma. A second portion was homogenised in phosphate buffered saline for Lowry total protein measurement. Results were expressed per $g$ of total protein.

\section{Plasma $\alpha$-tocopherol}

This was measured by normal phase HPLC using a Waters w600E pump and a Waters 490 fluorescence detector. Delta tocopherol was used as an internal standard. ${ }^{17}$

\section{Superoxide dismutase}

A further aliquot of the homogenised liver was incubated with a stock solution of nitroblue tetrazolium $(25 \mathrm{mM})$ and xanthine $(10 \mathrm{mM})$, to which was added xanthine oxidase $(2 \cdot 3$ $\mathrm{nM}$ ). The superoxide radical, generated by the oxidation of xanthine, was measured by its ability to reduce niroblue tetrazolium. Superoxide dismutase was quantitated in terms of its ability to inhibit this reaction. ${ }^{18}$

\section{STATISTICAL ANALYSIS}

Following an analysis of variance, after showing that a difference exists, the results were compared using unpaired $t$ tests. Significance was taken at the $5 \%$ level.

\section{Results}

The blood alcohol concentrations achieved with the gavage regime can be seen in Figure 1. Mean peak concentrations of $186 \mathrm{mg} \%$ were achieved in males and $156 \mathrm{mg} \%$ in females, although at night it can be seen that levels fell dramatically. Histologically, only minor fatty change was produced.

Since no differences existed between the sexes with regard to malonaldehyde or antioxidant measurements, data were pooled for the analyses. Hepatic total glutathione concentrations were increased in the alcohol treated group compared with controls $(\mathrm{p}<0.0001)$ (Fig 2). There was no difference in the ratios of oxidised to reduced glutathione between the groups. Hepatic malonaldehyde concentrations were increased in the alcoholic animals compared with controls $(p<0.001)$ (Fig 3 ). There was a close correlation between glutathione and malonaldehyde values $(r=0.6$, $\mathrm{p}<0.0001$ ) in both alcohol treated and control groups (Fig 4). There were no significant differences in the hepatic concentrations of vitamins $\mathrm{C}$ and $\mathrm{E}$, nor in the plasma concentrations of vitamin $C$. In addition, the hepatic 


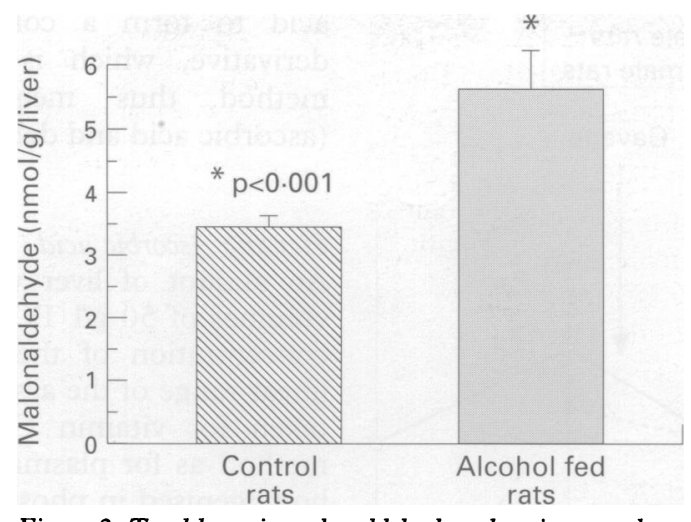

Figure 3: Total hepatic malonaldehyde values in control and alcohol fed rats (values, mean (SEM)).

superoxide dismutase activity also did not differ significantly (Table).

\section{Discussion}

The mammalian liver is effectively protected against both endogenous and exogenous oxidative stresses by intracellular glutathione via several enzymatic and non-enzymatically catalysed reactions. These include reduction of hydroperoxides by glutathione transferases, and handling of glutathione conjugates by $\gamma$ glutamyl transferases. ${ }^{19}$ In both $\operatorname{man}^{12}$ and primates, ${ }^{20}$ chronic alcohol feeding results in depressed hepatic reduced glutathione (GSH) and increased production of diene conjugates, evidence of lipid peroxidation. ${ }^{12}$ This was initially proposed by DiLuzio and Hartmann in $1967^{21}$ as an important mechanism in the pathogenesis of alcoholic liver disease, and considerable supporting

Antioxidants in liver and plasma (values, mean (SEM))

\begin{tabular}{lccl}
\hline & Alcoholic rats & Controls rats & Significance \\
\hline Plasma ascorbic acid (mmol//) & $44 \cdot 2(7 \cdot 8)$ & $50 \cdot 9(8 \cdot 6)$ & NS \\
Hepatic ascorbic acid (mmol/g protein) & $353 \cdot 8(26 \cdot 4)$ & $313 \cdot 8(13 \cdot 5)$ & NS \\
Plasma $\alpha$-tocopherol (mmol//) & $8 \cdot 1(1 \cdot 5)$ & $6 \cdot 2(1 \cdot 2)$ & NS \\
Hepatic superoxide dismutase (ng/g liver) & $0 \cdot 0462(0.003)$ & $0.048(0.004)$ & NS \\
\hline
\end{tabular}

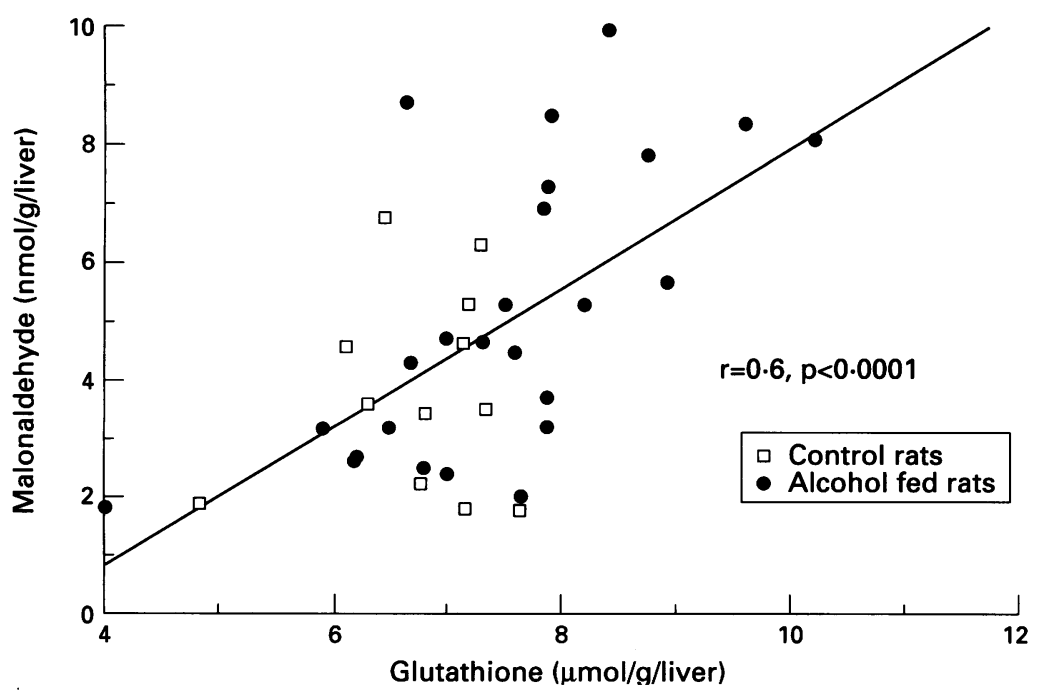

Figure 4: Correlation of malonaldehyde and glutathione concentrations in control and alcohol fed rats (values, mean (SEM)). evidence has since been reported. ${ }^{3} 112022-24$

The finding of a noticeable increase in hepatic glutathione is contrary to previous reports. ${ }^{12} \mathrm{GSH}$ synthesis is very active in the liver, which plays a major role in GSH metabolism. GSH effluxes from the liver into both the sinusoidal compartment and the bile, with the sinusoidal efflux being quantitatively the most important. ${ }^{25}$ After efflux from the liver, GSH is rapidly cleared from plasma by uptake into extrahepatic tissues. ${ }^{19}$ Lauterburg suggested that the low plasma levels of GSH that accompany fasting may be due to increased extrahepatic catabolism. Plasma measurements, however, are a poor indicator of the hepatic GSH pool, and are critically dependent on the nutritional state. ${ }^{26}$ Chronic ethanol feeding increases hepatic GSH turnover in the rat, ${ }^{27}$ and also increases the sinusoidal efflux of GSH, presumably as a direct effect on the liver rather than because of an increase in extrahepatic requirements.

The finding in our animals of increased hepatic glutathione may represent the nutritional status of the animals. All animals were killed over a two hour period on consecutive mornings, as we have previously shown that after a period of fasting, glutathione levels can rise by $45 \%$ in only 150 minutes (unpublished observations). The reduction of levels seen in other reports, ${ }^{3}$ particularly in man, ${ }^{12}$ may therefore represent the period of fasting undertaken before biopsy, or a fall in levels with progressive liver damage often present when human biopsy specimens are taken. Alternatively, this rise may be part of a protective mechanism preventing rodents from developing alcoholic liver damage, supported by the close correlation between hepatic glutathione and malonaldehyde values.

The levels of the plasma antioxidant $\alpha$-tocopherol were raised after chronic ethanol feeding, though this was not significant. There was no difference in the plasma or hepatic concentrations of ascorbic acid. Ethanol feeding reduces hepatic $\alpha$-tocopherol, ${ }^{23}$ with an increase in $\alpha$-tocopheryl quinone formation from free radical reactions. The increase in plasma $\alpha$-tocopherol may be related to increases in plasma lipids as a result of chronic ethanol feeding, since in plasma, $\alpha$-tocopherol is transported by low density lipoproteins the concentration of which is increased after ethanol feeding. Alternatively, when $\alpha$-tocopherol acts as a chain terminator, ascorbic acid can recycle the tocopheroxyl radical thus formed, replenishing the $\alpha$-tocopherol ${ }^{28}$ and forming semihydroascorbate. This is further oxidised to dehydroascorbate, which in turn is recycled to ascorbic acid using a dehydroascorbate reductase enzyme that utilises two molecules of glutathione. ${ }^{29}$ Thus, increased levels of glutathione allow the replenishment of both ascorbic acid and, subsequently, $\alpha$-tocopherol and may explain why in early alcoholic liver disease, before glutathione levels fall, these metabolites are present in normal concentrations.

Kamimura recently reported an association of lipid peroxidation with liver fibrogenesis, 
though they did not detect evidence for peroxidation before fibrosis developed. This is possibly since the measurement of 4 hydroxynonenal is less sensitive, since it is present in far lower concentrations. Detection of malonaldehyde by HPLC is both sensitive and specific, ${ }^{14}$ so the increase that we have shown is strong evidence for the role of peroxidation in the aetiopathogenesis of alcoholic liver damage.

Despite a prolonged period with high blood alcohol levels, our animals failed to develop progressive liver damage, in contrast to the intragastric infusion techniques. ${ }^{30}$ The two major differences between the two methodologies are the inclusion of a high fat content as corn oil, and a continuous high blood alcohol level. The exact reason for the importance of a high fat content remains unclear.

1 Teare JP, Carmichael AJ, Burnett F, Rake MO. Detection of antibodies to acetaldehyde-albumin conjugates in alcoholic liver disease. Alcohol 1993; 28: 11-16.

2 Ekstrom G, Ingelman-Sundberg M. Rat liver microsomal NADPH-supported oxidase activity and lipid peroxidation dependent on ethanol inducible cytochrome $\mathrm{P}_{450}$. Biochem Pharmacol 1989; 38: 1313-19.

3 Kamimura S, Gaal K, Britton RS, Bacon BR, Triadafilopoulos G, Tsukamato H. Increased 4-hydroxynonenal levels in experimental alcoholic liver disease: nonenal levels in experimental alcoholic liver disease: Association of lipid peroxidation

4 Situnayake RD, Crump BJ, Thurnham D, Davies JA, Gearty J, Davis M. Lipid peroxidation and hepatic antioxidants in alcoholic liver disease. Gut 1990; 31: 1311-17.

5 Cederbaum AI. Microsomal generation of hydroxyl radicals: Its role in microsomal ethanol oxidising system activity and its requirement for iron. Ann NY Acad Sci 1987; 492: 35-49.

6 Williams AJ, Barry RE. Free radical generation by neutrophils: A potential mechanism of cellular injury in acute alcoholic hepatitis. Gut 1987; 28: 1157-61.

7 Reinke LA, Rau JM, McCay PB. Possible roles of free radicals in alcoholic tissue damage. Free Radic Res Commun 1990; 9: 205-11.

8 Albano E, Tomasi A, Goria-Gatti L, Diazani MU. Sign trapping of free radical species produced during the microsomal metabolism of ethanol. Chem Biol Interact 1988; 65: 223-34.

9 Inomata T, Rao GA, Tsukamato $H$. Lack of evidence for increased lipid peroxidation in ethanol-induced centrilobular necrosis of rat liver. Liver 1987; 7: 233-9.

10 Speisky H, Bunout D, Orrego H, Israel Y. Lack of changes in diene conjugate levels following ethanol induced glutathione depletion or hepatic necrosis. Res Commun Chem Pathol Pharmacol 1985; 48: 77-90.
11 Strubelt O, Younes M, Pentz R. Enhancement by glutathione depletion of ethanol induced acute hepatotoxicity in-vitro and in-vivo. Toxicology 1987; 45: 213-23.

12 Shaw S, Rubin KP, Lieber CS. Depressed hepatic glutathione and increased zinc conjugates in alcoholic liver disease: Evidence of lipid peroxidation. Dig Dis Sci 1983; 7: 585-9.

13 Teare JP, Punchard NA, Powell JJ, Mitchell WD, Lumb PJ, Thompson RPH. An automated spectrophotometric method for the determination of oxidised and reduced glutathione in liver. Clin Chem 1993; 38: 686-9.

14 Esterbauer H, Lang J, Zadravec S, Slater TF. Detection of malonaldehyde by high performance liquid chromatography. In: Packer L, ed. Methods in enzymology. Orlando: graphy. In: Packer L, ed. Methods

15 Slater TF. In: Free radical mechanisms in tissue injury. London: Pion, 1972: 38.

16 Pesce AJ, Kaplan LA. Methods in clinical chemistry. St Louis: CV Mosby \& Co, 1987: 577.

17 Buttriss JL, Diplock AT. High performance liquid chromatography methods for vitamin $\mathrm{E}$ in tissues. In: Packer L, ed. Methods in enzymology. Orlando: Academic Press, 1984: 131-8.

18 McCord JM, Fridovich I. The reduction of cytochrome c by milk xanthine oxidase. F Biol Chem 1968; 243: 5733-60.

19 Wendel A, Akryt P. The level and half-life of glutathione in plasma. FEBS Lett 1980; 120: 209-11.

20 Shaw S, Jayatilleke E, Ross WA, Gordon E, Lieber CS. Ethanol induced lipid peroxidation: Potentiation by long term alcohol feeding and attenuation by methionine. 7 Lab Clin Med 1981; 98: 417-25.

21 Di Luzio N, Hartmann AD. Role off lipid peroxidation in the pathogenesis of the ethanol induced fatty liver. Fed Proc 1967; 26: 1436-42.

22 Albano E, Tomasi A, Goria-Gatti L, Poli G, V Annini V, Dianzani MU. Free radical metabolism of alcohols by rat liver mocrosomes. Free Radic Res Commun 1989; 3: 243-9.

23 Kawase T, Kato S, Lieber CS. Lipid peroxidation and antioxidant defense systems in rat liver after chronic ethanol feeding. Hepatology 1989; 10: 815-21.

24 Videla LA, Valenzuela A. Alcohol ingestion, liver glutathiome and lipoperoxidation: Metabolic inter-relations thiome and lipoperoxidation: Metabolic inter-relations and patho

25 Callans DJ, Walker LS, Mitchell MC. Effects of ethanol feeding and withdrawal on plasma glutathione elimination in the rat. Hepatology 1987; 7: 496-501.

26 Lauterburg BH, Adams JD, Mitchell JR. Hepatic glutathione homeostasis in the rat: efflux account for glutathione turnover. Hepatology 1984; 7: 586-90.

27 Morton S, Mitchell MC. Increased hepatic efflux of glutathione after chronic ethanol feeding. Biochem Pharmacol 1985; 34: 1559-63.

28 Buettner GR. The pecking order of free radicals and antioxidants: lipid peroxidation, $\alpha$-tocopherol and ascorbate. Arch Biochem Biophys 1993; 300: 535-43.

29 Meister A. On the antioxidant effects of ascorbic acid and glutathione. Biochem Pharmacol 1992; 10: 1905-15.

30 Tsukamato H, Gaal K, French SW. Insights into the pathogenesis of alcoholic liver necrosis and fibrosis: status report. Hepatology 1990; 12: 599-608. 